Nloman 2021, 39(1)

Revista de Psicologia, Ciències de l'Eduació i de l'Esport

ISSN: 1138-3194

Facultat de Psicologia, Ciències de l'Educació i de l'Esport Blanquerna

Universitat Ramon Llull

\title{
Uso problemático del smartphone: el papel de los Cinco Grandes, la Tríada Oscura y la impulsividad
}

\author{
Sergio Hidalgo-Fuentes \\ Universidad de Valencia
}

Recibido: 2020-11-27

Aceptado: 2021-3-21

doi: 10.51698/aloma.2021.39.1.17-26

Uso problemático del smartphone: el papel de los Cinco Grandes, la Tríada Oscura y la impulsividad

Resumen. Este estudio examinó las relaciones entre los rasgos de personalidad de los cinco grandes (extraversión, afabilidad, neuroticismo, responsabilidad y apertura a la experiencia), la tríada oscura (maquiavelismo, psicopatía y narcisismo), la impulsividad y el uso problemático del smartphone. Doscientos dos participantes (52.48\% hombres), con edades comprendidas entre los 18 y los 58 años, completaron el Ten-Item Personality Inventory, la Smartphone Addiction Scale, el Dirty Dozen y la escala de impulsividad de Plutchik. Los análisis de correlación mostraron que el uso problemático del smartphone estaba significativamente asociado a la extraversión, el neuroticismo, la impulsividad y todos los rasgos de la tríada oscura. Realizando un análisis de regresión jerárquica, controlando la edad y el sexo, se encontró que la extraversión, el neuroticismo, el maquiavelismo, el narcisismo y la impulsividad fueron predictores significativos del uso problemático del smartphone. Este estudio contribuye a la literatura que ha examinado la relación entre los factores de personalidad y el uso problemático del smartphone.

Palabras clave: uso problemático; smartphone; cinco grandes; tríada oscura; impulsividad

Problematic smartphone use: the role of the Big Five, the Dark Triad and impulsivity

Abstract. This study examines the relationships among the Big Five personality traits (extraversion, agreeableness, neuroticism, conscientiousness and openness to experience), the Dark Triad (Machiavellianism, psychopathy and narcissism), impulsivity and problematic smartphone use. A total of 202 participants (52.48\% male), ranging in age from 18 to 58 years old, completed the Smartphone Addiction Scale-Short Version, the Ten-Item Personality Inventory, the Dirty Dozen and the Plutchik Impulsivity Scale. Correlation analysis showed that problematic smartphone use was significantly correlated with extraversion, neuroticism, impulsivity and all the traits of the Dark Triad. Using hierarchical regression analyses, controlling for age and gender, it was found that extraversion, neuroticism, Machiavellianism, narcissism and impulsivity were significantly related to problematic smartphone use. This study contributes to the body of literature that has examined the relationship between personality traits and problematic smartphone use.

Keywords: problematic use; Smartphone; Big Five; Dark Triad; Impulsivity 


\section{Introducción}

El uso del smartphone está muy extendido en España; el 78.9\% de la población mayor de 15 años dispone de al menos un dispositivo y supera ampliamente la penetración de otros dispositivos digitales, como los ordenadores portátiles (57.8\%) o las tabletas (43.1\%) (ONTSI, 2019). De media, los españoles utilizan su smartphone durante casi dos horas y media diarias, tiempo que asciende a más de cuatro horas diarias en el caso de los jóvenes de entre 18 y 24 años, y se ha convertido en la opción preferida para realizar actividades como efectuar búsquedas, visitar las redes sociales o ver vídeos (Rivero, 2019).

A pesar de que numerosos estudios han investigado el abuso o la dependencia del smartphone como una adicción (Arnavut et al., 2018; Duke \& Montag, 2017; Jo et al., 2018), existe una controversia sobre la utilización del término adicción aplicado al uso excesivo de este dispositivo tecnológico (Panova \& Carbonell, 2018). Ni el DSM-5 (American Psychiatric Association, 2013) ni el CIE-11 (World Health Organization, 2018) recogen la adicción al smartphone, mientras que diversos estudios han encontrado un solapamiento entre la supuesta adicción al smartphone y el uso problemático de internet o de determinadas redes sociales y aplicaciones de mensajería instantánea (Kuss y Griffiths, 2017; Montag et al., 2019; Sha et al., 2019), con lo que han considerado el smartphone como un medio para acceder a estas últimas. Además, algunos autores señalan que las consecuencias negativas provocadas por la utilización excesiva del smartphone no son comparables a las causadas por otro tipo de adicciones, por lo que desaconsejan hablar de adicción y recomiendan expresiones alternativas, como uso problemático (Panova \& Carbonell, 2018). Debido a lo expuesto, en este estudio se utiliza la expresión uso problemático del smartphone, definido como el uso compulsivo del smartphone con repercusiones negativas en cuanto a productividad, relaciones sociales, salud física o bienestar emocional (Horwood \& Anglim, 2018). La prevalencia de este problema en España se ha estimado entre un $12.5 \%$ (Lopez-Fernandez, 2017) y un 23.67\% (Ballestar-Tarín et al., 2020); investigaciones similares en otros países mostraron prevalencias que van desde un $16.9 \%$ entre los jóvenes suizos (Haug et al., 2015) hasta un 30.9\% en los adolescentes surcoreanos (Cha \& Seo, 2018). El uso problemático del smartphone se asocia a un importante número de consecuencias negativas, como pérdida de la calidad de sueño (Demirci et al., 2015), lesiones cervicales (AlAbdulwahab et al., 2017), aumento del consumo de alcohol (Grant et al., 2019), estrés y ansiedad (Hawi \& Samaha, 2017; Vahedi \& Saiphoo, 2018), depresión (Elhai et al., 2017; Elhai et al., 2016), phubbing o ignorar a una persona durante una interacción social por centrarse en el smartphone (Chotpitayasunondh \& Douglas, 2016; Davey et al., 2018; Karadağ et al., 2015), bajo rendimiento académico (Amez \& Baert, 2020; Samaha \& Hawi, 2016), descenso de la productividad laboral (Duke \& Montag,
2017), miedo a que otros tengan experiencias gratificantes sin que uno esté presente o fear of missing out (Elhai et al., 2020; Gil et al., 2015; Wolniewicz et al., 2018), conflictos con familiares y amigos (Kim et al., 2019) e ideación suicida (Arrivillaga et al., 2020).

La personalidad se encuentra entre las variables que pueden contribuir en mayor medida al comportamiento adictivo (Grant et al., 2010). Una de las teorías de la personalidad más extendidas es el modelo de los cinco grandes, que incluye los factores de extraversión (ser sociable), afabilidad (ser tolerante), apertura a la experiencia (ser creativo), neuroticismo (ser emocionalmente inestable) y responsabilidad (ser organizado) (Costa \& McCrae, 1992). Un reciente metaanálisis que ha examinado los resultados de 36 estudios ha mostrado cómo el alto neuroticismo y la baja responsabilidad presentan asociaciones significativas con el uso problemático del smartphone (Marengo et al., 2020). Asimismo, el modelo teórico desarrollado por Billieux y colaboradores para explicar el uso problemático del teléfono móvil presenta el neuroticismo y la inestabilidad emocional como factores de riesgo que pueden provocar un patrón adictivo en cuanto al uso de este dispositivo (Billieux et al., 2015).

La tríada oscura es un conjunto de tres rasgos de personalidad desadaptativos: maquiavelismo, narcisismo subclínico y psicopatía subclínica (Paulhus \& Williams, 2002). El maquiavelismo consiste en la tendencia a manipular, explotar y utilizar a otras personas como un medio para conseguir los propios fines (Christie \& Geis, 1970). El narcisismo se caracteriza por un autoconcepto exagerado y poco realista, una firme creencia de tener derecho a privilegios y el deseo de ser admirado y respetado por los demás (Brummelman et al., 2016). La psicopatía se define como una combinación de impulsividad y tendencias antisociales, como falta de remordimientos, problemas para establecer vínculos emocionales o baja empatía (Lyons, 2019; Paulhus \& Williams, 2002). Una reciente revisión de la literatura científica ha mostrado que el narcisismo y la psicopatía se relacionan tanto con adicciones relacionadas con sustancias como no relacionadas con sustancias (Jauk \& Dieterich, 2019). Los mecanismos que favorecen la adicción en relación con la psicopatía y el narcisismo difieren, mientras que las dificultades en los procesos de autorregulación podrían fomentar comportamientos adictivos en los sujetos narcisistas (Salazar-Fraile et al., 2010), los individuos con rasgos psicopáticos podrían verse impulsados por la desinhibición asociada a estos (Jauk \& Dieterich, 2019). Sin embargo, son pocos los estudios que se han centrado en el papel de la tríada oscura en el uso problemático del smartphone de manera específica (Balta et al., 2019; Ksinan et al., 2019).

Por su parte, la impulsividad se puede definir como una tendencia a actuar rápidamente, antes de procesar completamente la información disponible y sin considerar adecuadamente las consecuencias a largo plazo (Moeller et al., 2001). La capacidad de controlar nuestros impulsos se ha mostrado fundamental en múltiples 
facetas tanto sociales como individuales, y su ausencia se asocia tanto a adicciones relacionadas con sustancias (Jakubczyk et al., 2018; Liu et al., 2019; Thomsen et al., 2018; Willhelm et al., 2020) como al uso problemático de Facebook (Cudo et al., 2020), Internet (Levi et al., 2020; Li et al., 2019; Liu et al., 2019), o el smartphone (Grant et al., 2019; Kim et al., 2016; Peterka-Bonetta et al., 2019).

Aunque sí que se han encontrado estudios que relacionan tanto los componentes de la tríada oscura como la impulsividad con el uso problemático de internet o de determinadas redes sociales (Facebook, Instagram...) o aplicaciones de mensajería (WhatsApp, Line...), existe una falta de investigaciones que se centren en el uso problemático del smartphone. En el caso de los factores de los cinco grandes, sí se ha encontrado un número importante de estudios en los que se investiga su asociación con el uso problemático del smartphone, aunque cuentan con limitaciones, como presentar en la mayoría de los casos una sobrerrepresentación de mujeres en las muestras seleccionadas (Marengo et al., 2020). Por ello, el presente estudio trata de estimar la contribución de los factores de los cinco grandes, la tríada oscura (maquiavelismo, narcisismo subclínico y psicopatía subclínica) y de la impulsividad en la predicción del uso problemático del smartphone. Se espera contrastar las siguientes hipótesis: H1) el neuroticismo y la baja responsabilidad serán predictores del uso problemático del smartphone; H2) el narcisismo y la psicopatía serán predictores del uso problemático del smartphone y; H3) la impulsividad será predictor del uso problemático del smartphone.

\section{Método}

\section{Participantes}

Antes de dar comienzo a la fase de recogida de datos, se realizó un análisis de potencia a priori mediante el programa estadístico G*Power 3.1 (Faul et al., 2009) con el objetivo de calcular el tamaño muestral. Asumiendo un tamaño del efecto de $.15, \alpha=.05$ y $1-\beta=$ .95 , la muestra mínima requerida fue de 178 participantes. Los participantes debían tener una edad superior a los 18 años y poseer un smartphone. Se reclutó a 202 participantes, 106 hombres y 96 mujeres, con edades comprendidas entre los 18 y los 58 años $(M=$ 24.73; $D T=6.90)$.

\section{Material}

Se elaboró un cuestionario mediante la plataforma Google Forms en el que se recogió información sociodemográfica (sexo y edad) y se incluyeron pruebas para evaluar el uso problemático del smartphone, los cinco grandes, la tríada oscura y la impulsividad.

\section{Smartphone Addiction Scale-Short Version (SAS-SV)}

El uso problemático del smartphone fue evaluado mediante la escala SAS-SV (Kwon et al., 2013a). Esta es la versión corta de la escala SAS, desarrollada para medir la adicción al smartphone (Kwon et al., 2013b). La escala está formada por diez ítems con formato de respuesta tipo Likert de seis puntos, desde 1 (totalmente en desacuerdo) a 6 (totalmente de acuerdo). La puntuación total oscila en un rango de 10 a 60, y las puntuaciones mayores se asocian con la máxima prevalencia de adicción al smartphone. Para este estudio se empleó la adaptación al español realizada por LopezFernandez (2017) y que presentó un valor alfa de Cronbach de .88 y una adecuada validez de contenido con respecto a la asociación con indicadores como días por semana que se utiliza el smartphone para realizar actividades de ocio en línea $(r=.49, p<001)$ o tiempo al día que se utiliza el smartphone para realizar actividades de ocio en línea $(r=.35, p<001)$. Además, los resultados obtenidos en la adaptación mediante el análisis factorial exploratorio presentaron un solo factor (uso excesivo del smartphone) que explicó el 49.3\% de la varianza total (Lopez-Fernandez, 2017).

\section{Ten-Item Personality Inventory (TIPI)}

Los cinco grandes de personalidad fueron evaluados con el Ten-Item Personality Inventory (Gosling et al., 2003). Se utilizó la adaptación española del TIPI (Romero et al., 2012). Esta prueba proporciona una medida de los factores de personalidad que forman el modelo de los cinco grandes (extraversión, afabilidad, responsabilidad, estabilidad emocional y apertura a la experiencia) mediante diez ítems compuestos por dos adjetivos cada uno en los que el sujeto debe marcar el grado en el que se ve representado a través de una escala Likert de siete puntos, desde 1 (totalmente en desacuerdo) hasta 7 (totalmente de acuerdo). Las distintas escalas de la adaptación española presentan unos valores alfa de Cronbach moderados entre .38 y .59 , una característica habitual en las escalas ultracortas (Ziegler et al., 2014). La prueba presenta una buena validez convergente con el NEO-PIR y con otras pruebas relevantes y el análisis exploratorio realizado durante la adaptación retuvo cinco factores que representaban el $69.72 \%$ de la varianza total (Romero et al., 2012).

\section{Dirty Dozen (DD)}

Para evaluar los factores pertenecientes a la tríada oscura se usó la escala Dirty Dozen (Jonason \& Webster, 2010). En este estudio se utilizó la versión española de la escala DD adaptada por Pineda, Sandín y Muris (2018). La escala DD es una medida de la tríada oscura (maquiavelismo, psicopatía y narcisismo) mediante doce ítems, cuatro para cada uno de los rasgos, que los participantes valoraron sobre una escala Likert de cinco puntos, desde 0 (totalmente en desacuerdo) a 4 (totalmente de acuerdo). A mayor puntuación, mayores niveles de los respectivos rasgos. La adaptación utilizada muestra unos valores alfa de Cronbach entre .60 y .81 para los diferentes rasgos, así como de .80 para la puntuación global. El análisis exploratorio presentó tres factores (narcisismo, maquiavelismo y psicopatía) que explicaban un $67.27 \%$ de la varianza total (Maneiro et al., 2019). 
Escala de impulsividad de Plutchik (EI)

La impulsividad fue medida mediante la escala de impulsividad de Plutchik (Plutchik \& Van Praag, 1989). Para el presente estudio se usó la adaptación española (Rubio et al., 1998). La EI es un cuestionario para medir el comportamiento impulsivo mediante quince ítems valorados sobre una escala de tipo Likert de cuatro puntos, desde 0 (nunca) a 3 (casi siempre). El rango de puntuaciones varía de 0 a 45 . Las puntuaciones más altas indican niveles superiores de impulsividad. La adaptación española utilizada presenta un valor alfa de Cronbach de .71 (Alcázar-Córcoles et al., 2017) y el análisis exploratorio extrajo cuatro factores que explicaban un $47.31 \%$ de la varianza total (Alcázar-Córcoles et al., 2015).

\section{Procedimiento}

Se publicaron hilos con invitaciones a participar en el estudio en diferentes foros de internet relacionados con la telefonía móvil y las nuevas tecnologías tras obtener el permiso de sus administradores o moderadores (adslzone.net, HTCMania.com...). Adicionalmente, también se informó mediante correo electrónico a estudiantes de una universidad pública de Valencia de la realización del estudio y se solicitó su participación. En ambos casos, las invitaciones a participar contenían información sobre los objetivos del estudio, su carácter anónimo y voluntario, y un enlace al cuestionario en línea creado mediante la herramienta Google Forms. Además, se instaba a los participantes a difundir el formulario entre sus contactos de las redes sociales. Una vez los participantes accedían al enlace del cuestionario, la primera página les presentaba información sobre el estudio en el que de nuevo se garantizaba el anonimato de los datos recogidos. Antes de comenzar a cumplimentar el cuestionario, los participantes debían dar su consentimiento a participar en el estudio mediante un ítem específico creado a tal efecto. No se ofrecieron incentivos por la participación en el estudio.

\section{Análisis de datos}

El análisis de los datos se realizó mediante el paquete estadístico IBM SPSS Statistics v.24. Se calcularon los estadísticos descriptivos y las correlaciones bivariadas entre las distintas variables objeto de estudio. Para valorar el tamaño del efecto entre hombres y mujeres se utilizó el estadístico $q$ de Cohen, que permite valorar la diferencia entre dos correlaciones, los valores comprendidos entre .1 y .3 representan un tamaño del efecto pequeño; entre .3 y .5 , medio; y los superiores a .5 , grande (Cohen, 1988).

Posteriormente, con el objetivo de valorar la capacidad predictiva de los rasgos de los cinco grandes, la tríada oscura y la impulsividad sobre el uso problemático del smartphone, se realizó un análisis de regresión jerárquica. Con el uso problemático del smartphone como variable dependiente, las variables sociodemográficas sexo y edad entraron en el primer paso; en el segundo paso se introdujeron los factores de personalidad pertenecientes a los cinco grandes, en el tercer paso se incluyeron los componentes de la tríada oscura y en el cuarto paso se introdujo la impulsividad.

\section{Resultados}

En la Tabla 1 se muestran los estadísticos descriptivos de las variables analizadas y el alfa de Cronbach de las escalas correspondientes y en la Tabla 2 se presentan las correlaciones bivariadas para todas las medidas utilizadas. Respecto a la muestra total y en relación con los cinco grandes, el uso problemático del smartphone mantiene correlaciones estadísticamente significativas con la extraversión de manera positiva y la estabilidad emocional de manera negativa. Los tres componentes de la tríada oscura, así como la impulsividad, presentan correlaciones positivas estadísticamente significativas con el uso problemático del smartphone. En los hombres, la extraversión no mantiene su correlación con el uso problemático del smartphone, pero aparece una correlación negativa con la responsabilidad. En las mujeres, la psicopatía no correlaciona con el uso problemático del smartphone, pero sí la afabilidad y la apertura a la experiencia, de manera negativa. Las mayores diferencias entre las correlaciones entre hombres y mujeres se dan en maquiavelismo, responsabilidad y afabilidad, con un tamaño del efecto medio, pues las dos primeras son mayores en los hombres y la tercera en las mujeres.

Se realizó un análisis de regresión jerárquica para valorar la capacidad predicha de los factores sociode-

Tabla 1. Estadísticos descriptivos de las variables objeto de estudio y alfa de Cronbach de las escalas utilizadas

\begin{tabular}{|c|c|c|c|c|c|c|c|}
\hline & \multicolumn{2}{|l|}{ Total } & \multicolumn{2}{|c|}{ Hombres } & \multicolumn{2}{|c|}{ Mujeres } & \multirow[b]{2}{*}{$\alpha$} \\
\hline & $\mathbf{M}$ & DT & M & DT & M & DT & \\
\hline 1. Uso problemático del smartphone & 29.59 & 11.54 & 24.42 & 10.30 & 35.30 & 10.10 & .91 \\
\hline 2. Extraversión & 4.09 & 1.70 & 3.51 & 1.56 & 4.74 & 1.62 & .87 \\
\hline 3. Afabilidad & 5.21 & 1.26 & 4.97 & 1.24 & 5.48 & 1.23 & .66 \\
\hline 4. Responsabilidad & 4.49 & 1.46 & 4.18 & 1.62 & 4.83 & 1.17 & .71 \\
\hline 5. Estabilidad emocional & 4.45 & 1.55 & 4.64 & 1.42 & 4.24 & 1.65 & .79 \\
\hline 6. Apertura a la experiencia & 5.08 & 1.11 & 4.86 & 1.16 & 5.31 & 1.00 & .49 \\
\hline 7. Maquiavelismo & 8.16 & 3.53 & 8.27 & 3.63 & 8.03 & 3.43 & .84 \\
\hline 8. Psicopatía & 7.67 & 2.59 & 7.76 & 2.70 & 7.57 & 2.47 & .54 \\
\hline 9. Narcisismo & 9.22 & 4.23 & 8.60 & 4.03 & 9.90 & 4.35 & .87 \\
\hline 10. Impulsividad & 34.76 & 7.43 & 33.04 & 7.17 & 36.66 & 7.28 & .86 \\
\hline
\end{tabular}


Tabla 2. Correlaciones bivariadas de las variables objeto de estudio y q de Cohen

\begin{tabular}{|c|c|c|c|c|}
\hline & \multicolumn{3}{|c|}{ Uso problemático del smartphone } & \multirow[b]{2}{*}{$q$} \\
\hline & Total & Hombres & Mujeres & \\
\hline Extraversión & $.298^{\star *}$ & .079 & $.237^{*}$ & -.16 \\
\hline Afabilidad & .047 & .080 & $-.215^{*}$ & .30 \\
\hline$\underline{\text { Responsabilidad }}$ & -.040 & $-.290^{\star *}$ & .015 & -.31 \\
\hline Estabilidad emocional & $-.437^{* *}$ & $-.391^{\star *}$ & $-.470^{\star *}$ & .10 \\
\hline Apertura a la experiencia & -.011 & -.043 & $-.231^{*}$ & .19 \\
\hline Maquiavelismo & $.306^{\star *}$ & $.216^{*}$ & $.544^{* *}$ & -.39 \\
\hline Psicopatía & $.186^{\star *}$ & $.260^{*}$ & .196 & .07 \\
\hline Narcisismo & $.505^{* *}$ & $.505^{* *}$ & $.490^{* *}$ & .02 \\
\hline Impulsividad & $.543^{\star *}$ & $.526^{* *}$ & $.472^{* *}$ & .07 \\
\hline
\end{tabular}

mográficos, los cinco grandes, la tríada oscura y la impulsividad sobre el uso problemático del smartphone. Sexo (hombres $=1$, mujeres $=2$ ) y edad se introdujeron en el primer paso del análisis para controlar sus efectos, los componentes de los cinco grandes entraron en el segundo paso, los componentes de la tríada oscura fueron introducidos en el tercer paso y la impulsividad entró en el cuarto paso. Los resultados de este análisis se presentan en la Tabla 3. Previamente se verificaron los supuestos de linealidad, mediante los diagramas de dispersión parcial para cada variable independiente, homocedasticidad con la prueba de Levene, normalidad de los residuos con la prueba de Kolmogoroff-Smirnov, independencia de los residuos mediante el estadístico de Durbin-Watson y se descartó la multicolinealidad, al mostrarse todos los valores de inflación de varianza inferiores a 2.4 y los índices de tolerancia superiores a 0.4 . El primer paso del modelo explica un $23 \%$ de la varianza del uso problemático del smartphone, y el sexo es un predictor significativo $(\beta=.429 ; p<.001)$. Con la inclusión de los componentes pertenecientes a los cinco grandes, la varianza del uso problemático del smartphone explicada sube hasta el 39.6\%, y la extraversión $(\beta=.176 ; p<.01)$ y la estabilidad emocional $(\beta$ $=-.335 ; p<.001)$ son predictores estadísticamente significativos. Al añadir los rasgos de la tríada oscura se obtiene un incremento significativo de la varianza explicada, que alcanza el 50.8\%, y el maquiavelismo ( $\beta=.157 ; p<.05)$ y el narcisismo $(\beta=.236 ; p<.01)$ se muestran como predictores significativos del uso problemático del smartphone. El último paso aumenta la varianza explicada un $3.4 \%$, y la impulsividad es un predictor significativo $(\beta=.281 ; p<.001)$. El modelo global explica un $54.2 \%$ de la varianza asociada al uso problemático del smartphone y todos los pasos añaden una mejora significativa al ajuste del modelo $(p<.001)$.

\section{Discusión}

La Organización Mundial de la Salud ha afirmado que el uso excesivo de internet, así como de los dispositivos digitales entre los que se encuentran los smartphone ha alcanzado la magnitud de problema de salud público (World Health Organization, 2015). El objetivo de esta investigación era analizar la capacidad predictiva que los factores de personalidad pertenecientes a los modelos de los cinco grandes, los componentes de la tríada oscura y la impulsividad tienen sobre el uso problemático del smartphone. El análisis de regresión jerárquica muestra cómo la extraversión, la estabilidad emocional, el maquiavelismo, el narcisismo y la impulsividad predicen el uso problemático del smartphone más allá de la varianza explicada por las variables de control de edad y sexo.

Entre las variables sociodemográficas de control, se encontró un efecto significativo del sexo, pues el hecho de ser mujer se muestra como un predictor del uso problemático del smartphone, lo que coincide con diversos trabajos previos (Arnavut et al., 2018; Veissière $\&$ Stendel, 2018). Investigaciones anteriores han hallado que las mujeres hacen un uso fundamentalmente social del teléfono móvil, y dedican más tiempo a las aplicaciones relacionadas con la mensajería instantánea y las redes sociales (Carbonell et al., 2018); mientras que en los hombres los patrones de uso son más diversos (De-Sola et al., 2016).

Tabla 3. Regresión múltiple jerárquica: contribución de variables sociodemográficas (paso 1), los cinco grandes (paso 2), la tríada oscura (paso 3) y la impulsividad (paso 4) al uso problemático del smartphone

\begin{tabular}{|c|c|c|c|c|c|c|c|}
\hline \multirow{2}{*}{$\begin{array}{l}\text { Uso problemático del } \\
\text { smartphone }\end{array}$} & \multirow[b]{2}{*}{ B } & \multicolumn{2}{|c|}{ 95\% IC para B } & \multirow[b]{2}{*}{ SE } & \multirow[b]{2}{*}{$\beta$} & \multirow[b]{2}{*}{$\mathbf{R}^{2}$} & \multirow[b]{2}{*}{$\Delta \mathbf{R}^{2}$} \\
\hline & & LI & LS & & & & \\
\hline Modelo 1 & & & & & & $.230^{* * *}$ & \\
\hline Edad & -.163 & -.391 & .066 & .116 & -.097 & & \\
\hline Sexo & $9.887^{* * *}$ & 6.735 & 13.040 & 1.599 & $.429^{\star * *}$ & & \\
\hline Modelo 2 & & & & & & $.396^{* * *}$ & $.166^{* \star \star}$ \\
\hline Extraversión & $1.194^{\star *}$ & .314 & 2.073 & .446 & $.176^{\star *}$ & & \\
\hline Afabilidad & .076 & -.989 & 1.141 & .540 & .008 & & \\
\hline Responsabilidad & -.551 & -1.480 & .379 & .471 & -.070 & & \\
\hline Estabilidad emocional & $-2.507^{* * *}$ & -3.396 & -1.618 & .451 & $-.335^{\star * *}$ & & \\
\hline Apertura a la experiencia & -.998 & -2.314 & .318 & .667 & -.096 & & \\
\hline Modelo 3 & & & & & & $.508^{\star * \star}$ & $.112^{\star \star \star}$ \\
\hline Maquiavelismo & $.512^{*}$ & .078 & .946 & .220 & $.157^{\star}$ & & \\
\hline Psicopatía & .363 & -.171 & .896 & .271 & .081 & & \\
\hline Narcisismo & $.644^{* *}$ & .274 & 1.013 & .188 & $.236^{* *}$ & & \\
\hline Modelo 4 & & & & & & $.542^{\star * *}$ & $.034^{\star * \star}$ \\
\hline Impulsividad & $.436^{* * *}$ & .207 & .665 & .116 & $.281^{* * *}$ & & \\
\hline
\end{tabular}


En cuanto a los factores de personalidad pertenecientes a los cinco grandes, la H1 encuentra apoyo parcial, ya que la baja estabilidad emocional se presenta como predictor del uso problemático del smartphone, pero no así la baja responsabilidad. El hallazgo de que la baja estabilidad emocional se asocie de manera positiva y sea un predictor significativo del uso problemático del smartphone es congruente con la literatura previa sobre el tema (Cho et al., 2017; Horwood \& Anglim, 2018; Marengo et al., 2020; Peterka-Bonetta et al., 2019). Las personas más inestables emocionalmente podrían tener un riesgo superior de presentar un uso problemático del smartphone al tratar de controlar el estatus de sus relaciones sociales comprobando de manera compulsiva las redes sociales y las aplicaciones de mensajería (Billieux, 2012). En cambio, el hecho de que la extraversión resulte un predictor significativo del uso problemático del smartphone no coincide con el reciente metaanálisis de Marengo y sus colaboradores (2020), que no encuentran correlación significativa entre este rasgo de personalidad y el uso problemático del smartphone. Por el contrario, se ha hipotetizado que la extraversión podría ser un factor de riesgo al uso problemático del smartphone al hacer sentir un intenso y constante deseo de estar permanentemente en contacto con otras personas y establecer nuevas relaciones (Billieux et al., 2015). Esto estaría en consonancia con el hecho de que el uso excesivo de aplicaciones, como WhatsApp, se relacione con el uso problemático del smartphone (Sha et al., 2019).

En relación con los rasgos de la tríada oscura, tanto el narcisismo como el maquiavelismo se muestran como predictores significativos del uso problemático del smartphone. El hecho de que el narcisismo prediga el uso problemático del smartphone es coherente con lo encontrado en investigaciones anteriores (Balta et al., 2019; Pearson y Hussain, 2015). Los individuos con altos niveles de narcisismo podrían utilizar diferentes redes sociales a través del smartphone para promocionarse a sí mismos y satisfacer sus necesidades de atención y admiración (Casale \& Fioravanti, 2018; Kircaburun \& Griffiths, 2018). Por el contrario, que el maquiavelismo prediga de manera directa el uso problemático del smartphone no está en consonancia con los escasos estudios que investigan la relación entre la tríada oscura y el uso de este dispositivo (Balta et al., 2019). Sí que hay evidencias de que el maquiavelismo se relaciona con un incremento de la utilización de aplicaciones relacionadas con las compras en línea (Burtăverde et al., 2019), así como con las apuestas en línea (Kircaburun \& Griffiths, 2018). Al ser el maquiavelismo un rasgo relacionado con el deseo de alcanzar un estatus social elevado (Dahling et al., 2008), las personas con un alto grado podrían utilizar este tipo de aplicaciones para tratar de conseguir objetivos relacionados con la posibilidad de mostrar a los demás una imagen de riqueza y poder. Por tanto, la H2 se cumple parcialmente al hallarse el narcisismo un predictor significativo del uso problemático del smartphone pero no encontrarse esta relación con la psicopatía.
Por último, se cumple la H3, ya que la impulsividad también se presenta como un predictor significativo del uso problemático del smartphone. Este resultado está en línea con la literatura previa sobre el tema (Grant et al., 2019; Jo et al., 2018; Kim et al., 2016; PeterkaBonetta et al., 2019; Wu et al., 2013). Además, este resultado era esperable, ya que la impulsividad se ha relacionado con numerosas adicciones, incluida la adicción a la cocaína (García-Marchena et al., 2018), al alcohol (Shin et al., 2012), a la comida (VanderBroekStice et al., 2017), al tabaco (Liu et al., 2019), al sexo (Levi et al., 2020), etc.

A pesar de los hallazgos encontrados, el presente estudio no se halla exento de limitaciones. En primer lugar, al ser una muestra no probabilística, los resultados podrían no ser totalmente generalizables. En segundo lugar, el hecho de tratarse de un diseño de tipo transversal impide comprobar la evolución de las variables a lo largo del tiempo, por lo que sería recomendable llevar a cabo estudios longitudinales. En tercer lugar, el uso de una escala ultracorta como el Ten-Item Personality Inventory podría generar dudas, a pesar de ello, es una escala validada y ampliamente utilizada en investigación, por lo que no se considera un problema. Por último, el uso problemático del smartphone se evalúa mediante una prueba de autoinforme, por lo que en futuros estudios sería interesante utilizar medidas complementarias, como evaluaciones conductuales objetivas del nivel de uso del smartphone o evaluaciones realizadas por parte de psicólogos. Además, también sería conveniente para futuras investigaciones valorar el tipo de uso que hacen los sujetos del smartphone (redes sociales, académico, mensajería instantánea, etc.).

A pesar de estas limitaciones, el presente estudio proporciona información relevante y novedosa sobre la relación de la personalidad con el uso problemático del smartphone que puede tener implicaciones en la comprensión teórica de este fenómeno.

Declaración de divulgación del autor. No existen intereses en conflicto.

\section{Referencias}

AlAbdulwahab, S. S., Kachanathu, S. J., \& AlMotairi, M. S. (2017). Smartphone use addiction can cause neck disability. Musculoskeletal Care, 15(1), 10-12. https://doi.org/10.1002/msc.1170

Alcázar-Córcoles, M. Á., Verdejo, A. J., \& Bouso-Sáiz, J. C. (2015). Propiedades psicométricas de la escala de impulsividad de Plutchik en una muestra de jóvenes hispanohablantes. Actas españolas de psiquiatría, 43(5), 161-169. https://doi.org/10.1016/j. apj.2017.02.002

Alcázar-Córcoles, M. Á., Verdejo-García, A., Bouso-Sáiz, J. C., Revuelta-Menéndez, J., \& Ramírez-Lira, E. (2017). Propiedades psicométricas del cuestionario de personalidad EPQ-A en una muestra de adolescentes hispanohablantes. Anuario de Psicología Jurídica, 27(1), 5156. https://doi.org/10.1016/j.apj.2017.02.002 
American Psychiatric Association. (2013). Diagnostic and statistical manual of mental disorders: DSM-5. American Psychiatric Publishing

Amez, S., \& Baert, S. (2020). Smartphone use and academic performance: A literature review. International Journal of Educational Research, 103, 101618. https:// doi.org/10.1016/j.ijer.2020.101618

Arnavut, A., Nuri, C., \& Direktor, C. (2018). Examination of the relationship between phone usage and smartphone addiction based on certain variables. Anales De Psicología / Annals of Psychology, 34(3), 446450. https://doi.org/10.6018/analesps.34.3.321351

Arrivillaga, C., Rey, L., \& Extremera, N. (2020). Adolescents' problematic internet and smartphone use is related to suicide ideation: Does emotional intelligence make a difference?. Computers in Human Behavior, 110, 106375. https://doi.org/10.1016/j. chb.2020.106375

Balta, S., Jonason, P., Denes, A., Emirtekin, E., Tosuntas, S. B., Kircaburun, K., \& Griffiths, M. D. (2019). Dark personality traits and problematic smartphone use: The mediating role of fearful attachment. Personality and Individual Differences, 149, 214-219. https://doi. org/10.1016/j.paid.2019.06.005

Ballestar-Tarín, M. L., Simó-Sanz, C., Chover-Sierra, E., Saus-Ortega, C., Casal-Angulo, C., \& Martínez-Sabater, A. (2020). Self-Perception of dependence as an indicator of smartphone addiction-Establishment of a cutoff point in the SPAI-Spain Inventory. International Journal of Environmental Research and Public Health, 17(11), 3838. https://doi.org/10.3390/ijerph17113838

Billieux, J. (2012). Problematic use of the mobile phone: A literature review and a pathways model. Current Psychiatry Reviews, 8(4), 299-307. https://doi. org/10.2174/157340012803520522

Billieux, J., Maurage, P., Lopez-Fernandez, O., Kuss, D. J., \& Griffiths, M. D. (2015). Can disordered mobile phone use be considered a behavioral addiction? An update on current evidence and a comprehensive model for future research. Current Addiction Reports, 2(2), 156-162. https://doi.org/10.1007/s40429-0150054-y

Brummelman, E., Thomaes, S., \& Sedikides, C. (2016). Separating narcissism from self-esteem. Current Directions in Psychological Science, 25(1), 8-13. https:// doi.org/10.1177/0963721415619737

Burtăverde, V., Vlăsceanu, S., \& Avram, E. (2019). Exploring the relationship between personality structure and smartphone usage. Current Psychology, 1-13. https://doi.org/10.1007/s12144-019-00521-5

Carbonell, X., Chamarro, A., Oberst, U., Rodrigo, B., \& Prades, M. (2018). Problematic use of the Internet and smartphones in university students: 2006-2017. International Journal of Environmental Research and Public Health, 15(3), 1-13. https://doi.org/10.20944/ preprints201801.0236.v1

Casale, S., \& Fioravanti, G. (2018). Why narcissists are at risk for developing Facebook addiction: The need to be admired and the need to belong. Addictive Be- haviors, 76, 312-318. https://doi.org/10.1016/j. addbeh.2017.08.038

Cha, S. S., \& Seo, B. K. (2018). Smartphone use and smartphone addiction in middle school students in Korea: Prevalence, social networking service, and game use. Health psychology open, 5(1), 1-15. https:// doi.org/10.1177/2055102918755046

Cho, H.-Y., Kim, D. J., \& Park, J. W. (2017). Stress and adult smartphone addiction: Mediation by selfcontrol, neuroticism, and extraversion. Stress and Health, 33(5), 624-630. https://doi.org/10.1002/ smi. 2749

Chotpitayasunondh, V., \& Douglas, K. M. (2016). How "phubbing" becomes the norm: The antecedents and consequences of snubbing via smartphone. Computers in Human Behavior, 63, 9-18. https://doi. org/10.1016/j.chb.2016.05.018

Christie, R., \& Geis, F. (1970). Studies in Machiavellianism. Academic Press.

Cohen, J. (1988). Statistical Power Analysis for the Behavioral Sciences. Lawrence Erlbaum Associates.

Costa, P. T., Jr., \& McCrae, R. R. (1992). Revised NEO Personality Inventory (NEO-PI-R) and NEO Five Factor Inventory (NEO-FFI) professional manual. Psychological Assessment Resources.

Cudo, A., Torój, M., Demczuk, M., \& Francuz, P. (2020). Dysfunction of self-control in Facebook addiction: Impulsivity is the key. Psychiatric Quarterly, 91(1), 91101. https://doi.org/10.1007/s11126-019-09683-8

Dahling, J. J., Whitaker, B. G., \& Levy, P. E. (2008). The development and validation of a new machiavellianism scale. Journal of Management, 35(2), 219-257. https://doi.org/10.1177/0149206308318618

Davey, S., Davey, A., Raghav, S. K., Singh, J. V., Singh, N., Blachnio, A., \& Przepiórkaa, A. (2018). Predictors and consequences of "Phubbing" among adolescents and youth in India: An impact evaluation study. Journal of family \& community medicine, 25(1), 35-42. https://doi.org/10.4103/jfcm.JFCM_71_17

Demirci, K., Akgönül, M., \& Akpinar, A. (2015). Relationship of smartphone use severity with sleep quality, depression, and anxiety in university students. Journal of behavioral addictions, 4(2), 85-92. https:// doi.org/10.1556/2006.4.2015.010

De-Sola, J., Rodríguez, F., \& Rubio, G. (2016). Cellphone addiction: A review. Frontiers in psychiatry, 7, 175. https://doi.org/10.3389/fpsyt.2016.00175

Duke, É., \& Montag, C. (2017). Smartphone addiction, daily interruptions and self-reported productivity. Addictive Behaviors Reports, 6, 90-95. https://doi. org/10.1016/j.abrep.2017.07.002

Elhai, J. D., Dvorak, R. D., Levine, J. C., \& Hall, B. J. (2017). Problematic smartphone use: A conceptual overview and systematic review of relations with anxiety and depression psychopathology. Journal of affective disorders, 207, 251-259. https://doi. org/10.1016/j.jad.2016.08.030

Elhai, J. D., Gallinari, E. F., Rozgonjuk, D., \& Yang, H. (2020). Depression, anxiety and fear of missing out as correlates of social, non-social and problematic 
smartphone use. Addictive behaviors, 105, 106335. https://doi.org/10.1016/j.addbeh.2020.106335

Elhai, J. D., Levine, J. C., Dvorak, R. D., \& Hall, B. J. (2016). Fear of missing out, need for touch, anxiety and depression are related to problematic smartphone use. Computers in Human Behavior, 63, 509-516. https://doi.org/10.1016/j.chb.2016.05.079

Faul, F., Erdfelder, E., Buchner, A., \& Lang, A.-G. (2009). Statistical power analyses using G*Power 3.1: Tests for correlation and regression analyses. Behavior Research Methods, 41, 1149-1160.

García-Marchena, N., Ladrón de Guevara-Miranda, D., Pedraz, M., Araos, P. F., Rubio, G., Ruiz, J. J., Pavón, F. J., Serrano, A., Castilla-Ortega, E., Santín, L. J., \& Rodríguez de Fonseca, F. (2018). Higher impulsivity as a distinctive trait of severe cocaine addiction among individuals treated for cocaine or alcohol use disorders. Frontiers in Psychiatry, 9, 26 https://doi. org/10.3389/fpsyt.2018.00026

Gil, F., Oberst, U., Del Valle, G., \& Chamarro, A. (2015). Nuevas tecnologías - ¿Nuevas patologías? El Smartphone y el fear of missing out. Aloma: Revista de Psicologia, Ciències de l'Educació i de l'Esport, 33(2), 77-83. https:// doi.org/10.51698/aloma.2015.33.2.77-83

Gosling, S. D., Rentfrow, P. J., \& Swann, W. B. (2003). A very brief measure of the Big-Five personality domains. Journal of Research in personality, 37(6), 504-528. https://doi.org/10.1016/s0092-6566(03)00046-1

Grant, J. E., Lust, K., \& Chamberlain, S. R. (2019). Problematic smartphone use associated with greater alcohol consumption, mental health issues, poorer academic performance, and impulsivity. Journal of behavioral addictions, 8(2), 335-342. https://doi. org/10.1556/2006.8.2019.32

Grant, J. E., Potenza, M. N., Weinstein, A., \& Gorelick, D. A. (2010). Introduction to behavioral addictions. The American journal of drug and alcohol abuse, 36(5), 233-241. https://doi.org/10.3109/00952990.2010.4 91884

Hawi, N. S., \& Samaha, M. (2017). Relationships among smartphone addiction, anxiety, and family relations. Behaviour \& Information Technology, 36(10), 10461052 . https://doi.org/10.1080/014492 9x.2017.1336254

Haug, S., Castro, R. P., Kwon, M., Filler, A., Kowatsch, T., \& Schaub, M. P. (2015). Smartphone use and smartphone addiction among young people in Switzerland. Journal of behavioral addictions, 4(4), 299-307. https://doi.org/10.1556/2006.4.2015.037

Horwood, S., \& Anglim, J. (2018). Personality and problematic smartphone use: A facet-level analysis using the Five Factor Model and HEXACO frameworks. Computers in Human Behavior, 85, 349-359. https://doi.org/10.1016/j.chb.2018.04.013

Jakubczyk, A., Trucco, E. M., Kopera, M., Kobyliński, P., Suszek, H., Fudalej, S., Brower, K. J., \& Wojnar, M. (2018). The association between impulsivity, emotion regulation, and symptoms of alcohol use disorder. Journal of substance abuse treatment, 91, 49-56. https:// doi.org/10.1016/j.jsat.2018.05.004
Jauk, E., \& Dieterich, R. (2019). Addiction and the Dark Triad of Personality. Frontiers in psychiatry, 10, 662. https://doi.org/10.3389/fpsyt.2019.00662

Jo, H. S., Na, E., \& Kim, D. J. (2018). The relationship between smartphone addiction predisposition and impulsivity among Korean smartphone users. Addiction Research \& Theory, 26(1), 77-84. https://doi.org/ 10.1080/16066359.2017.1312356

Jonason, P. K., \& Webster, G. D. (2010). The dirty dozen: a concise measure of the dark triad. Psychological Assessment, 22(2), 420-432. https://doi.org/10.1037/ a0019265

Karadă̆, E., Tosuntaš, S.B., Erzen, E., Duru, P., Bostan, N., Sahin, B.M., Çulha, İ., \& Babadă̆, B. (2015). Determinants of phubbing, which is the sum of many virtual addictions: A structural equation model. Journal of behavioral addictions, 4(2), 60-74. https:// doi.org/10.1556/2006.4.2015.005

Kim, Y., Jeong, J. E., Cho, H., Jung, D. J., Kwak, M., Rho, M. J., Yu, H., Kim, D. J., \& Choi, I. Y. (2016). Personality factors predicting smartphone addiction predisposition: Behavioral inhibition and activation systems, impulsivity, and self-control. PloS one, 11(8), e0159788. https://doi.org/10.1371/journal. pone.0159788

Kim, M. H., Min, S., Ahn, J. S., An, C., \& Lee, J. (2019). Association between high adolescent smartphone use and academic impairment, conflicts with family members or friends, and suicide attempts. PloS one, 14(7), e0219831. https://doi.org/10.1371/journal. pone.0219831

Kircaburun, K., \& Griffiths, M. D. (2018). The dark side of internet: Preliminary evidence for the associations of dark personality traits with specific online activities and problematic internet use. Journal of Behavioral Addictions, 7(4), 993-1003. https://doi. org/10.1556/2006.7.2018.109

Ksinan, A. J., Mališ, J., \& Vazsonyi, A. T. (2019). Swiping away the moments that make up a dull day: Narcissism, boredom, and compulsive smartphone use. Current Psychology, 1-10. https://doi.org/10.1007/ s12144-019-00228-7

Kuss, D. J., \& Griffiths, M. D. (2017). Social networking sites and addiction: Ten lessons learned. International journal of environmental research and public health, 14(3), 311. doi:10.3390/ijerph14030311

Kwon, M., Kim, D. J., Cho, H., \& Yang, S. (2013a). The smartphone addiction scale: development and validation of a short version for adolescents. PloS one, 8(12), e83558. https://doi.org/10.1371/journal. pone.0083558

Kwon, M., Lee, J. Y., Won, W. Y., Park, J. W., Min, J. A., Hahn, C., Gu, X., Choi, J. Y., \& Kim, D. J. (2013b). Development and validation of a smartphone addiction scale (SAS). PloS one, 8(2), e56936. https://doi. org/10.1371/journal.pone.0056936

Levi, G., Cohen, C., Kaliche, S., Sharaabi, S., Cohen, K., Tzur-Bitan, D., \& Weinstein, A. (2020). Sexual addiction, compulsivity, and impulsivity among a predominantly female sample of adults who use the 
internet for sex. Journal of Behavioral Addictions, 9(1), 83-92. https://doi.org/10.1556/2006.2020.00007

Li, Q., Dai, W., Zhong, Y., Wang, L., Dai, B., \& Liu, X. (2019). The mediating role of coping styles on impulsivity, behavioral inhibition/approach system, and internet addiction in adolescents from a gender perspective. Frontiers in Psychology, 10, 2402. https:// doi.org/10.3389/fpsyg.2019.02402

Liu, S. J., Lan, Y., Wu, L., \& Yan, W. S. (2019). Profiles of impulsivity in problematic internet users and cigarette smokers. Frontiers in psychology, 10, 772. https://doi.org/10.3389/fpsyg.2019.00772

Lopez-Fernandez, O. (2017). Short version of the Smartphone Addiction Scale adapted to Spanish and French: Towards a cross-cultural research in problematic mobile phone use. Addictive behaviors, 64, 275280. https://doi.org/10.1016/j.addbeh.2015.11.013

Lyons, M. (2019). The Dark Triad of personality: Narcissism, Machiavellianism, and psychopathy in everyday life. Academic Press.

Maneiro, L., López-Romero, L., Gómez-Fraguela, J. A., Cutrín, O., \& Romero, E. (2019). Pursuing the Dark Triad: Psychometric properties of the Spanish version of the Dirty Dozen. Journal of Individual Differences, 40(1), 36. https://doi.org/10.1027/1614-0001/ a000274

Marengo, D., Sindermann, C., Häckel, D., Settanni, M., Elhai, J. D., \& Montag, C. (2020). The association between the Big Five personality traits and smartphone use disorder: A meta-analysis. Journal of Behavioral Addictions, 9(3), 534-550. https://doi. org/10.1556/2006.2020.00069

Moeller, F. G., Barratt, E. S., Dougherty, D. M., Schmitz, J. M., \& Swann, A. C. (2001). Psychiatric aspects of impulsivity. American journal of psychiatry, 158(11), 1783-1793. https://doi.org/10.1176/appi. ajp.158.11.1783

Montag, C., Wegmann, E., Sariyska, R., Demetrovics, Z., \& Brand, M. (2019). How to overcome taxonomical problems in the study of Internet use disorders and what to do with "smartphone addiction"? Journal of behavioral addictions, 1-7. https://doi. org/10.1556/2006.8.2019.59

ONTSI. (2019). La sociedad en red. Transformación digital en España. Informe Anual 2018. ONTSI

Panova, T., \& Carbonell, X. (2018). Is smartphone addiction really an addiction? Journal of Behavioral Addictions, 7(2), 252-259.

Paulhus, D. L., \& Williams, K. M. (2002). The dark triad of personality: Narcissism, Machiavellianism, and psychopathy. Journal of research in personality, 36(6), 556-563. https://doi.org/10.1016/s00926566(02)00505-6

Pearson, C., \& Hussain, Z. (2015). Smartphone use, addiction,narcissism, and personality: A mixed methods investigation. International Journal of Cyber Behavior, Psychology and Learning, 5(1), 17-32. https:// doi.org/10.4018/ijcbpl.2015010102

Peterka-Bonetta, J., Sindermann, C., Elhai, J. D., \& Montag, C. (2019). Personality associations with smartphone and internet use disorder: A comparison study including links to impulsivity and social anxiety. Frontiers in public health, 7, 127. https://doi. org/10.3389/fpubh.2019.00127

Pineda, D., Sandín, B., \& Muris, P. (2018). Psychometrics properties of the spanish version of two dark triad scales: The dirty dozen and the short dark triad. Current Psychology, 1-9. https://doi.org/10.1007/ s12144-018-9888-5

Plutchik, R., \& Van Praag, H. M. (1989). The measurement of suicidality, aggressivity and impulsivity. Progress in Neuro-Psychopharmacology \& Biological Psychiatry, 13, 23-24.

Rivero, F. (2019) Informe ditrendia: Mobile en España y en el Mundo 2018. Ditrendia.

Romero, E., Villar, P., Gómez-Fraguela, J. A., \& LopezRomero, L. (2012). Measuring personality traits with ultra-short scales: A study of the Ten Item Personality Inventory (TIPI) in a Spanish sample. Personality and Individual Differences, 53(3), 289-293. https://doi. org/10.1016/j.paid.2012.03.035

Rubio, G., Montero, I., Jáuregui, J., Martínez, M. L., Álvarez, S., Marín, J. J., \& Santo-Domingo, J. (1998). Validación de la escala de impulsividad de Plutchik en población española. Archivos de Neurobiología, 61(3), 223-232.

Salazar-Fraile, J., Ripoll-Alandes, C., \& Bobes, J. (2010). Open narcissism, covered narcissism and personality disorders as predictive factors of treatment response in an out-patient Drug Addiction Unit. Adicciones, 22(2). 107-112.

Samaha, M., \& Hawi, N. S. (2016). Relationships among smartphone addiction, stress, academic performance, and satisfaction with life. Computers in Human Behavior, 57, 321-325. https://doi.org/10.1016/j. chb.2015.12.045

Sha, P., Sariyska, R., Riedl, R., Lachmann, B., \& Montag, C. (2019). Linking internet communication and smartphone use disorder by taking a closer look at the Facebook and WhatsApp applications. Addictive behaviors reports, 9, 100148. https://doi.org/10.1016/j. abrep.2018.100148

Shin, S. H., Hong, H. G., \& Jeon, S.-M. (2012). Personality and alcohol use: The role of impulsivity. Addictive Behaviors, 37(1), 102-107. https://doi. org/10.1016/j.addbeh.2011.09.006

Thomsen, K. R. , Callesen, M. B., Hesse, M, Kvamme, T. L., Pedersen, M. M., Pedersen, M. U., \& Voon, V. (2018). Impulsivity traits and addiction-related behaviors in youth. Journal of behavioral addictions, 7(2), 317-330. https://doi.org/10.1556/2006.7.2018.22

Vahedi, Z., \& Saiphoo, A. (2018). The association between smartphone use, stress, and anxiety: A metaanalytic review. Stress and Health, 34(3), 347-358. https://doi.org/10.1002/smi.2805

VanderBroek-Stice, L., Stojek, M. K., Beach, S. R. H., vanDellen, M. R., \& MacKillop, J. (2017). Multidimensional assessment of impulsivity in relation to obesity and food addiction. Appetite, 112, 59-68. https://doi.org/10.1016/j.appet.2017.01.009 
Veissière, S. P., \& Stendel, M. (2018). Hypernatural monitoring: A social rehearsal account of smartphone addiction. Frontiers in Psychology, 9, 141. https:// doi.org/10.3389/fpsyg.2018.01118

Willhelm, A. R., Pereira, A. S., Czermainski, F. R., Nogueira, M., Levandowski, D. G., Volpato, R. B., \& de Almeida, R. M. M. (2020). Aggressiveness, impulsiveness, and the use of alcohol and drugs: Understanding adolescence in different contexts. Trends in Psychology, 28(3), 381-398. https://doi.org/10.1007/ s43076-020-00022-6

Wolniewicz, C. A., Tiamiyu, M. F., Weeks, J. W., \& Elhai, J. D. (2018). Problematic smartphone use and relations with negative affect, fear of missing out, and fear of negative and positive evaluation. Psychiatry research, 262, 618-623. https://doi.org/10.1016/j. psychres.2017.09.058
World Health Organization. (2015). Public health implications ofexcessive use of the Internet, computers, smartphones and similar electronic devices. https://apps. who.int/iris/handle/10665/184264

World Health Organization. (2018). International Classification of Diseases 11th Revision (ICD-11). https:// icd.who.int/

Wu, A. M. S., Cheung, V. I., Ku, L., \& Hung, E. P. W. (2013). Psychological risk factors of addiction to social networking sites among Chinese smartphone users. Journal of Behavioral Addictions, 2(3), 160-166. https://doi.org/10.1556/jba.2.2013.006

Ziegler, M., Kemper, C. J., \& Kruyen, P. (2014). Short Scales - Five Misunderstandings and Ways to Overcome Them. Journal of Individual Differences, 35(4), 185-189. https://doi.org/10.1027/1614-0001/ a000148 